\title{
THEORETICAL REMARKS ON FERROMAGNETISM AT LOW TEMPERATURES
}

\author{
BY \\ L. NEEL \\ UNIVERSITÉ, GRENoble
}

Conrents: 1. Introduction, 336. - 2. Finely Dispersed Substances, 337. 3. Substances with Bloch Walls, 340. -4. Thermal Activation, 341.

\section{Introduction}

Among the questions in the domain of ferromagnetism which may be solved by using low temperatures, those that are directly concerned with the thermal fluctuations appear to be the most interesting: their manifestation depends on the ratio of the thermal energy $k T$ to the various anisotropy energies, and this ratio can be varied between wide limits. Unfortunately, the properties involved must be studied in magnetic fields that are smaller than, or comparable to, the coercive force, i.e. in a region where the theoretical description of the phenomena does not yet possess the complete rigour and generality one would desire. No rapid progress can therefore be expected in this field.

There are only two extreme examples for which it is possible to give a clear and satisfactory picture of the magnetization process inside the hysteresis region. On the one hand there is the case of a finely divided substance the grains of which are so small that they contain only a single elementary Weiss domain, and which are sufficiently separated from each other, so that their interactions may be neglected. The other case is that of a solid substance which is sufficiently pure and homogeneous, so that the elementary domains are large and well-formed, and such that the walls that separate them (the Bloch walls) are approximately plane and at a distance from each other that is large compared to their thickness. Incidentally, pure iron is about the only substance for which it will be possible to satisfy these various conditions.

Let us investigate the importance of the thermal fluctuations in these two cases successively. 


\section{Finely Dispersed Substances}

One may either have independent grains, which are usually obtained by chemical means, or ferromagnetic grains that are dispersed in a non-magnetic phase, and which are obtained during segregation by cooling a homogeneous phase that is stable at high temperatures. If the grains are to contain only a single elementary domain, we have shown ${ }^{1}$ that their diameter must be smaller than a certain critical diameter $D_{c}$ given by:

$$
D_{c}=0.69 a N^{\frac{1}{2}}\left(\log \frac{D_{c}}{a}-0.307\right)^{\frac{1}{2}},
$$

in which $N$ is the coefficient of the molecular field (referred to unit volume) and $a$ the distance between neighbouring atoms: for iron, one thus finds $D_{c}=320 \AA$. For the ordinary ferromagnetic substances, neither $a$ nor $N$ varies appreciably between room temperature and absolute zero. We may therefore assume that the critical diameter $D_{c}$ is practically independent of the temperature.

When the diameter is smaller than $D_{c}$, the magnetic moment $M$ of a grain of volume $V$ preserves a constant magnitude equal to the product of the spontaneous magnetization $J$ and the volume $V$. The energy of the grain, on the other hand, depends on the orientation of $M$. Consequently, various factors come into play: the magnetocrystalline and the magnetoelastic energy, the demagnetizing field, and finally the surface anisotropy; a new effect which; as we have shown ${ }^{2}$, is of importance for the case of very small grains with a diameter smaller than $100 \AA$. The result is that in each grain the magnetization $M$ has two antiparallel orientations of minimum energy separated by a potential barrier. In the absence of an external magnetic field, the magnetic moment of a grain points in one of these two directions. When the magnetizing field has been reduced to zero after magnetization to saturation in a certain direction $\Delta$, the individual magnetic moments of the grains point in that direction of minimum energy that is closest to $\Delta$ : an ensemble of randomly oriented grains thus shows a remanent magnetization equal to one half of the saturation magnetization. To destroy this remanent magnetization, it is necessary to force the individual magnetic moments of the grains over the potential barriers by applying a certain coercive field $H_{c}$ in the opposite direction. This field is equal ${ }^{1}$ to $0.48 H_{o}$, if $H_{c}$ is the coercive force of an ensemble of grains for which the directions of minimum energy are all parallel 
to the direction of magnetization. This field is a measure for the height of the potential barrier separating the two antiparallel directions of minimum energy. Indicating the difference in energy between the top and the minimum of the barrier by $E$, we have ${ }^{3}$ :

$$
E=\frac{1}{2} V J_{s} H_{c} \text {. }
$$

The details of the theory of the magnetic properties of an ensemble of small grains has been the object of a number of papers '1' ${ }^{\prime}$ ' 4 , and it is unnecessary to reproduce them here. However, it is essential to remember that the theory is applicable only if the thermal energy $k T$ is considerably smaller than the energy $E$ defined above: to fix ideas, $k T$ must be 20 to 30 times smaller than $E$. Otherwise, the thermal fluctuations are able to force the magnetic moment of a grain over the potential barrier from one direction of minimum energy to the opposite one. In the limit of very small grains with a diameter of say $10 \AA$, in zero external field a time interval smaller than one millionth of a second is sufficient to go from one equilibrium position to the other. In other words, this ensemble of very small grains can reach the state of thermodynamical equilibrium that corresponds to the imposed external conditions almost instantaneously, and so behaves in first approximation like an ensemble of independent magnetic moments, subjected to the classical statistics, and of individual moment equal to $V J_{s}$ : there is no longer any hysteresis. Consequently, the ensemble of grains is paramagnetic, and no longer possesses any ferromagnetic properties, at least in so far as one considers these properties to be bound to the presence of a remanent magnetization and coercive force.

Hence it is necessary to mark the division between the ferro and the paramagnetic grains.

For this it is necessary to know the perturbing forces, or more exactly the perturbing couples perpendicular to $M$, which are able to turn the magnetic moment of a grain from one direction to the other. A complete list of these couples has not yet been made, but it appears that the couples corresponding to the variations in the demagnetizing field and the magnetocrystalline forces that result from the thermoelastic deformations of the crystal lattice, are the most effective.

Consider a grain with a magnetic symmetry of an ellipsoid of revolution, which is subjected to a magnetic field in the direction of the symmetry axis $R$. In the absence of any perturbations the magnetic moment $M$ describes a'precession around $R$, keeping the angle $\varphi$ 
between $M$ and $R$ constant. A perturbing couple $\Gamma$, perpendicular to $R$, brings about a change in $\psi$, and can thus overcome a potential barrier for $M$. In this way, the spontaneous thermoelastic deformations of the grain alter the symmetry and give rise to a perturbing couple of magnetocrystalline origin. The magnitude of the average value of this couple is given by ${ }^{3}$ the expression:

$$
\bar{\Gamma}=3 \lambda(2 G k T / \pi V)^{\frac{1}{2}} .
$$

These same thermoelastic deformations destroy the axial symmetry of the external form of the grain, and thus give rise to a perturbing demagnetizing field, and hence to a couple:

$$
\bar{\Gamma}=D J_{s}{ }^{2}(2 k T / \pi V G)^{\frac{1}{2}} \text {. }
$$

In these expressions, $\lambda$ is the longitudinal magnetostriction at saturation (supposed to be isotropic), $G$ is the modulus of shear elasticity, $k$ the Boltzmann constant, and $D$ a numerical coefficient depending on the form of the grain, but being always approximately equal to 3 . For small values of the volume $V$ of the grains, these perturbing couples are, of course, more important than for large values of $V$. Let us now consider an ensemble of identical grains, which at the time $t=0$ has a certain resultant remanent magnetization $M_{o}$ in an external field zero. As a result of the spontaneous reorientations of the magnetic moments, the resultant remanent moment decreases with increasing time, and at the time $t$ its value $M_{r}$ is given by:

$$
M_{r}=M_{o} \exp \left(-t / \tau_{o}\right) \text {, }
$$

in which $\tau_{o}$ is a relaxation time that can be expressed in terms of the perturbing couples by means of the relation ${ }^{3}$ :

$$
\frac{1}{\tau_{o}}=\left(e H_{c} \mid m\right)\left|3 G \lambda+D J_{s}{ }^{2}\right|\left(\frac{2 V}{\pi G k T}\right)^{\frac{1}{2}} \exp \left(-\frac{V H_{c} J_{s}}{2 k T}\right),
$$

where $e$ and $m$ represent respectively the elementary electric charge and the mass of the electron.

The application of this formula to grains of iron with a coercive force $H_{c}=1000$ oersteds shows that the relaxation time varies from $10^{-1}$ to $10^{9}$ seconds when the ratio $V / T$ increases from 3.2 tot 7.0 , which corresponds to an increase of the diameter of the grains of only $30 \%$.

If the relaxation time of a grain is smaller than $10^{-1}$ second, the grain may be said to be paramagnetic, since it comes to equilibrium in the applied field almost instantaneously. If, on the other hand, the 
relaxation time is larger than $10^{9}$ seconds, the grain must be said to be ferromagnetic, since it is able to preserve its remanent magnetization during a practically indefinite time. The numerical values given above show that in an ensemble of grains with different diameters, the division between the two kinds of grains is unambiguous and the transition region relatively small. For iron at room temperature this division corresponds to grains of a diameter of $160 \AA$, while at $1^{\circ} \mathrm{K}$ it would correspond to a diameter of $25 \AA$. By extending the study of the magnetic properties to very low temperatures, it is thus possible to explore the granular structure between the indicated limits.

In spite of the fact that the transition region between the paraand ferromagnetic grains is narrow, it is of importance that the magnetic moment of the grains belonging to the transition region, which may constitute 1 to $10 \%$ of the total mass, is a function of the time: there is magnetic relaxation. The investigation of this relaxation as a function of the temperature furnishes another means of studying the granular structure. The theory of this relaxation is complicated by the fact that the time constants that govern the rotations of the individual magnetic moments of the grains from one direction of minimum energy to the other, depend on the magnetic field to which the grains are subjected ${ }^{3}$.

\section{Substances with Bloch walls}

It is possible to give a satisfactory description of the influence of the thermal fluctuations on the propagation of the walls that separate the various elementary domains without entering into the details of the exact mechanism of the phenomenon, to which we will return later: we assume ${ }^{5}$ that there is a fluctuating field $H_{i}(t)$, which is an alternating function of the time with an average value equal to zero, which is superimposed algebraically on the external magnetic field applied to the sample. If the substance does not show any hysteresis, a small field of average value zero will not change the average magnetization of the substance. If there is hysteresis, however, this is no longer true: it depends on the magnetic history of the sample, whether the susceptibility in the direction considered will be equal to $a$ or to $a+c$, where $a$ and $c$ represent respectively the reversible and irreversible differential susceptibilities: $c$ depends in fact not only on the field and the magnetization but also on the magnetic history of the sample. The change in the magnetization resulting from the presence 
of the alternating field is equal to $c H_{i}{ }^{\prime}(t)$, where $H_{i}{ }^{\prime}(t)$ is the largest value attained by $H_{i}(t)$ in the chosen direction up till the time $t$.

This value $H_{i}{ }^{\prime}(t)$ increases of course with time, and one can show that under sufficiently general conditions $H_{i}{ }^{\prime}(t)$ is given by the expression

$$
H_{i}^{\prime}(t)=S(Q+\log t)
$$

in which $S$ is a constant depending on the nature of the substance and on the temperature, and in which $Q$ is a numerical constant of the order of 40 or 50 . In particular it follows that if one subjects a ferromagnetic substance to a magnetic field $H$, the substance behaves as if one had applied a field $H+S(Q+\log t)$.

The amplitude of the magnetic after-effect thus obtained, which we shall call the irreversible after-effect or, better, the thermal aftereffect, depends on the value of the constant $S$ which has the dimension of a magnetic field: the experiments show that the order of magnitude of $S$ is equal to one hundredth or one thousandth of the coercive force ${ }^{7,8}$.

There are several mechanisms which can give rise to the fluctuating field $H_{i}(t)$. The most effective one seems to arise from the fluctuations in the internal field that are produced by the thermal ondulations of the spinwaves, i.e. by the thermal oscillations of the clementary moments: it is, in fact, the "fluctuating" aspect of this same mechanism that is responsible for the decrease of the spontaneous magnetization under the influence of an increase in the temperature. A simple calculation shows ${ }^{5}$ that.

$$
S=r(k T / V)^{\frac{1}{2}}
$$

where $r$ is a numerical coefficient of the order of 0.3 , and $V$ the volume affected by an elementary Barkhausen discontinuity. In strongly perturbed materials, as in good permanent magnets, $V$ must be nearly equal to the volume of an elementary Weiss domain; in very soft materials on the contrary, $V$ must be considerably smaller than the volume of the elementary domains.

The study of the thermal variation of $S$ is of great interest and may yield information about the possible dependence of the volume of the Barkhausen discontinuities and the elementary domains on the temperature.

\section{Thermal Activation}

The thermal fuctuations, or more exactly the thermal activation, may 
be of importance also for the magnetic relaxation due to diffusion. The best known example is constituted by the interstitial carbon atoms in the crystal lattice of $\alpha$ iron. The carbon atoms can occupy a number of different kinds of sites which are characterized by different energies that depend on the orientation of the spontaneous magnetization relative to the crystal axes, and which are such that a change in the magnetization must be accompanied by a redistribution in the positions of the carbon atoms. This mechanism gives rise to magnetic relaxation and to the disaccomodation of the permeability, as soon as the time during which the variations in the magnetization are produced is of the same order of magnitude as the time constant that characterizes the redistribution of the carbon atoms. This time constant is of the order of magnitude of $\exp (W / k T)$, where $W$ is the height of the potential barrier separating two possible positions of the interstitial atoms. This time constant is therefore extremely sensitive to variations in the temperature. The result is that the relaxation produced by the diffusion of atoms of a definite kind is observable only in a relatively small interval of temperature. Thus the band in carbon iron has a breadth of some hundred degrees and is centered around room temperature.

Besides carbon, the atoms of the elements oxygen, boron, etc., which are able to take up interstitial position in the iron lattice, are known to give rise to "diffusion" relaxation. We must further remark that the ferromagnetic substitution alloys are also able to give rise to diffusion relaxation: as we have shown in a recent paper ${ }^{3}$, the relative positions of the atoms $\mathrm{A}$ and $\mathrm{B}$ in the state of equilibrium of a binary alloy $\mathrm{AB}$ depend on the orientation of the spontaneous magnetization. However, in this case the activation energy $W$ is probably much larger than in the interstitial alloys, so that the relaxation band must be shifted to higher temperatures, for the ferro-nickels for instance to about $700^{\circ} \mathrm{K}$. The application of low temperatures is therefore of no importance for the study of this question, except in the case of magnetite or substances with a similar structure, which can be considered to be composed of ferrous and ferric ions. These ions can play the role of different atoms, but the interchange of a ferrous and a ferric ion does not require the interchange of the complete atoms but only the simple transfer of an electron from one atom to the other. The activation energy is then much smaller, and the corresponding relaxation band is probably located at about $100^{\circ} \mathrm{K}$ : interesting problems can then arise in the domain of low temperatures. 
THEORETICAL REMARKS ON FERROMAGNETISM AT LOW TEMP. 343

\section{REFERENCES}

1 L. Néel, Comptes-rendus Ac. Sc., 224, 1488, 1550 (1947).

L. Neel, J. Physique et Radium, 15, 225 (1954).

${ }^{3}$ L. Néel, Ann. Géophysique, 5, 99 (1949).

4 E. C. Stoner and E. P. Wohlfahrt, Phil. Trans., 240, 599 (1948).

5 L. Neel, J. Physique et Radium, 11, 49 (1950).

- L. Néel, J. Physique et Radium, 12, 339 (1951).

7 J. C. Barbier, Ann. Phys., 9, 84 (1954).

${ }^{8}$ L. Lliboutry, Ann. Phys., 6, 731 (1951).

${ }^{9}$ J. L. Snoek, Physica, 5, 663 (1938). 